\title{
Model Systems Facilitating an Understanding of Mechanisms for Oncogene Amplification
}

Takaaki Watanabe

Additional information is available at the end of the chapter

http://dx.doi.org/10.5772/55119

\section{Introduction}

Gene amplification is a copy number increase of a restricted region of a chromosome arm. Amplified chromosomal regions are present in acentric mini extra-chromosome (double minutes, DMs) or within a chromosome as repetitive arrays (homogeneously staining regions, HSRs); or distributed at various locations in the genome (scattered-type) (Fig. 1).

Normal diploid genome
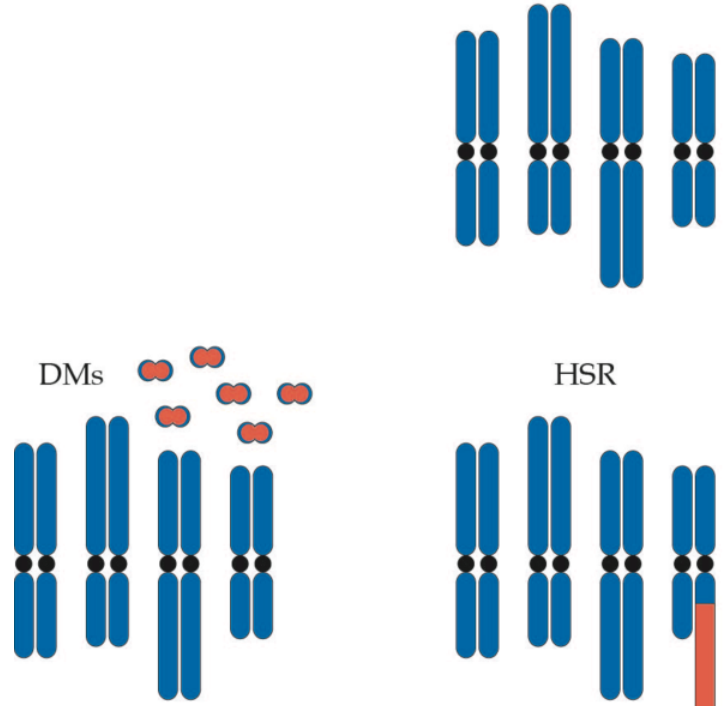

HSR

Scattered
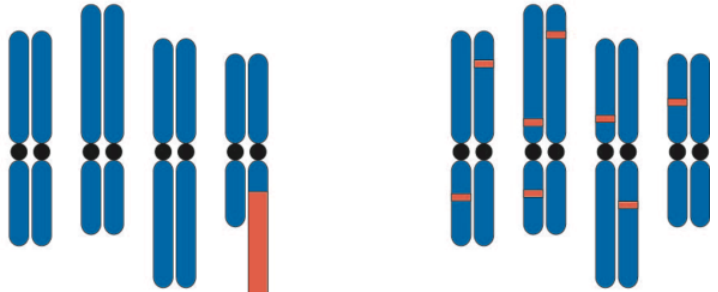

Figure 1. Typical amplification products in mammalian cells. 
A schematic illustration of four-chromosome genome (above) and three types of amplification products (below) are depicted. Amplified regions are indicated in red, and black circles represent centromeres. See text for details.

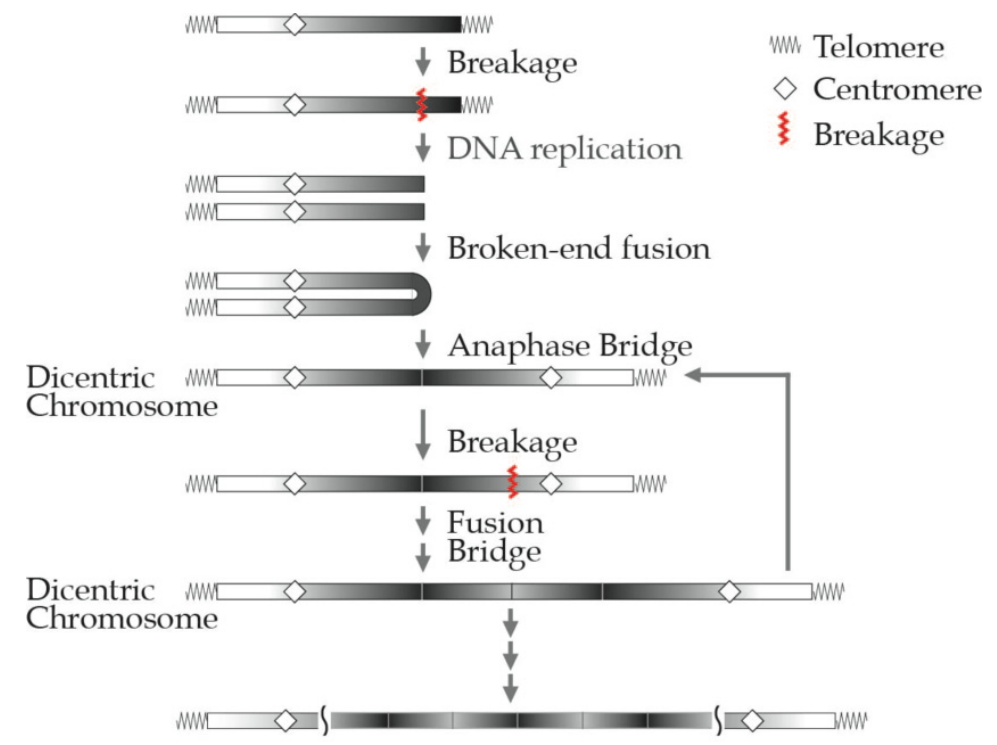

Figure 2. Breakage-fusion-bridge (BFB) cycles

The BFB cycle can be initiated by a DNA double-strand break. After DNA replication, the ends of the sister chromatids can fuse, giving rise to a dicentric chromosome. At anaphase, if the two centromeres go to the two opposite poles of the mitotic spindle, the dicentric chromosome can be broken. An asymmetric break will lead to a formation partially deleted or duplicated broken chromosomes. Subsequent cycles involving the chromosome with the duplication cause the increase in the copy number of the region of interest as inverted repeats. BFB cycles end when the broken chromosome ends are stabilized.

Oncogene amplification is common in human cancers and contributes to tumor progression and therapeutic resistance (Albertson, 2006; Tanaka and Yao, 2009). For example, ERBB2 amplification is often detected in advanced breast cancers, and overproduction of ERBB2 can accelerate tumor progression (Di Fiore et al., 1987; Muller et al., 1988; Slamon et al., 1987). Amplifications of MYC, CCND1, EGFR, MDM2, MYCN, JUN, TNK2, or ESR1 are also associated with aggressive phenotypes of tumors. BCR-ABL fusion gene is amplified in patients showing therapeutic resistance to Imatinib mesylate (Gorre et al., 2001). Amplifications of DHFR, TYMS, or MET are also associated with therapeutic resistance.

A variety of models are proposed to explain the amplification process, including unequal sister-chromatid exchange, localized over replication, fold-back priming, rolling-circle replication, and breakage-fusion-bridge (BFB) cycle (Kobayashi et al., 2004; McClintock, 1941; Rattray et al., 2005; Tower, 2004; Watanabe and Horiuchi, 2005). Cytogenetic features 
of BFB cycle have been repeatedly observed in tumor cells (Fig.2). BFB cycle is the most popular model to explain intra-chromosomal amplification (Mondello et al., 2010; Tanaka and Yao, 2009), especially in the early stage of the amplification. In cancer cells, HSRs are often organized as an inverted ladder associated with a deletion that spans from the amplicon toward a telomere (Debatisse and Malfor, 2005). According to the BFB model, such a complex rearrangement results from the following repeating cycle: an initial DSB; replication of the broken molecule; fusion of sister chromatids; formation of a bridge during anaphase; and asymmetrical breakage due to mechanical tension, which generates one chromatid with an inverted repeat at the broken end.

In extra-chromosomal amplification, replication-based models are often proposed. Breakage at stalled replication forks is proposed to cause DMs formation including EGFR gene (Vogt et al., 2004). Extra-rounds of replication are thought to lead to DMs containing N-myc gene. In N-myc amplification, extra-round of replication is expected to form an extrachromosomal element leading to integration followed by intra-chromosomal amplification (Savelyeva and Schwab, 2001). However, only by these amplification models themselves, it is difficult to explain the entire processes of the amplification.

\section{Barrier to efficient analyses}

Despite their biological and clinical importance, mechanisms for amplifying oncogenes remain largely unknown. This is because the whole process of gene amplification has been difficult to analyze because of additional kinds of amplification processes and secondary chromosome rearrangements (Haber and Debatisse, 2006). There are at least three reasons for this difficulty: (1) previous approaches to understand mechanisms for amplification were based on the structural analysis of complex end products; (2) few model systems for gene amplification are available that allow chromosomal engineering, as is possible in yeast (Lengauer et al., 1998); (3) spontaneous gene amplification occurs at very low frequency.

The use of genome-wide scanning techniques, such as array comparative genomic hybridization (array CGH) and next-generation DNA sequencing, has recently demonstrated that most solid tumors contain amplified portions of their genomes (Albertson, 2006). However, even these recent genomic technologies cannot unambiguously assign sequences in amplified regions and accurately resolve their chromosomal structure. Thus, amplified regions have been largely refractory to standard human genetic analyses.

\section{Model systems for understanding common features of gene amplification}

\subsection{DSB and inverted repeats}

Long series of studies have shown that DNA double-strand break (DSB) and inverted repeats play an important role in gene amplification. DNA double-strand break (DSB) is one of the harmful forms of DNA damage, and can induce several types of chromosomal aberrations, including gene amplification, when not correctly repaired. Amplification is 
triggered by DNA-damaging agents, which can directly or indirectly cause DSBs (Kuo et al., 1994; Paulson et al., 1998; Poupon et al., 1996; Yunis et al., 1987). In mammalian genomes, there are regions prone to breakage known as common fragile sites (CFSs) (Debatisse et al., 2012; Glover et al., 2005). CFSs are involved in chromosomal aberrations, including gene amplification, and have been shown to play a major role in the early steps in gene amplification (Ciullo et al., 2002; Coquelle et al., 1997; Hellman et al., 2002; Kuo et al., 1998). In cooperation with DSBs, short inverted repeats could generate a palindromic dicentric chromosome, leading to gene amplification.

Model systems that use site-specific endonucleases, such as I-SceI or HO endonucleases, have been constructed in yeast and in mammalian cells. Yao's group first constructed a plasmid-based system in yeast containing an $\mathrm{HO}$ endonuclease cutting site and an adjacent inverted repeat (Butler et al., 1996). This system efficiently formed a palindromic minichromosome after induction of the endonuclease. They next used Chinese hamster ovary $(\mathrm{CHO})$ cells and inserted a DHFR transgene into a chromosome of the cells with an I-Scel cutting site and an adjacent inverted repeat (Tanaka et al., 2002). This system formed a palindromic dimer after I-Scel cutting and consequently caused intra-chromosomal amplification, suggesting the formation of a dicentric chromosome and the involvement of subsequent BFB cycles.

We developed a new approach in which we design amplification processes and test whether the processes can produce the amplification seen in nature. Previously, in yeast, we constructed a system designed to induce a rapid amplification mode, double rolling-circle replication (DRCR) via chromosomal breaks induced by $\mathrm{HO}$ endonuclease (Watanabe and Horiuchi, 2005) (Fig.3). DRCR is a continuous process in which two replication forks chase each other (Fig.3A), and was first confirmed by Volkert and Broach for amplification of yeast $2 \mu$ plasmid (Volkert and Broach, 1986). To induce DRCR, we used break-induced replication (BIR), a nonreciprocal recombination-dependent replication process that is an effective mechanism to repair a broken chromosome (Fig.3B). The DRCR amplification is selected with an amplification marker, leu2d, which has a slight transcription activity and complements leucine auxotrophy if amplified (Erhart and Hollenberg, 1983). This system produced intra-/extra-chromosomal products resembling HSR and DMs seen in mammalian cells (Fig.3C). The HSR-type products contain up to $\sim 100$ copies of leu $2 d$ gene, which occupies $730 \mathrm{~kb}$ (the rest of chromosome VI comprises $275 \mathrm{~kb}$ ). Interestingly, HSR/DMs products were generated at low frequency without deliberate DNA cleavage, depending on the chromosome structure with the inverted repeats. These features strongly suggest that the processes described here may contribute to natural gene amplification in higher eukaryotes and natural amplification involves DRCR.

Lobachev et al constructed a yeast strain having an inverted repeat of Alu sequences, and showed that the repeat are fragile sites (Lobachev et al., 2002). The Alu inverted repeats can be cleaved and subsequently generate hairpin ends, which can be opened up by the Mre11/Rad50/Xrs2 complex in concert with the Sae2 protein. His group next demonstrated that Alu inverted repeats can trigger intra- and extra-chromosomal amplification in yeast (Narayanan et al., 2006). 
A

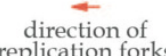
replication forks
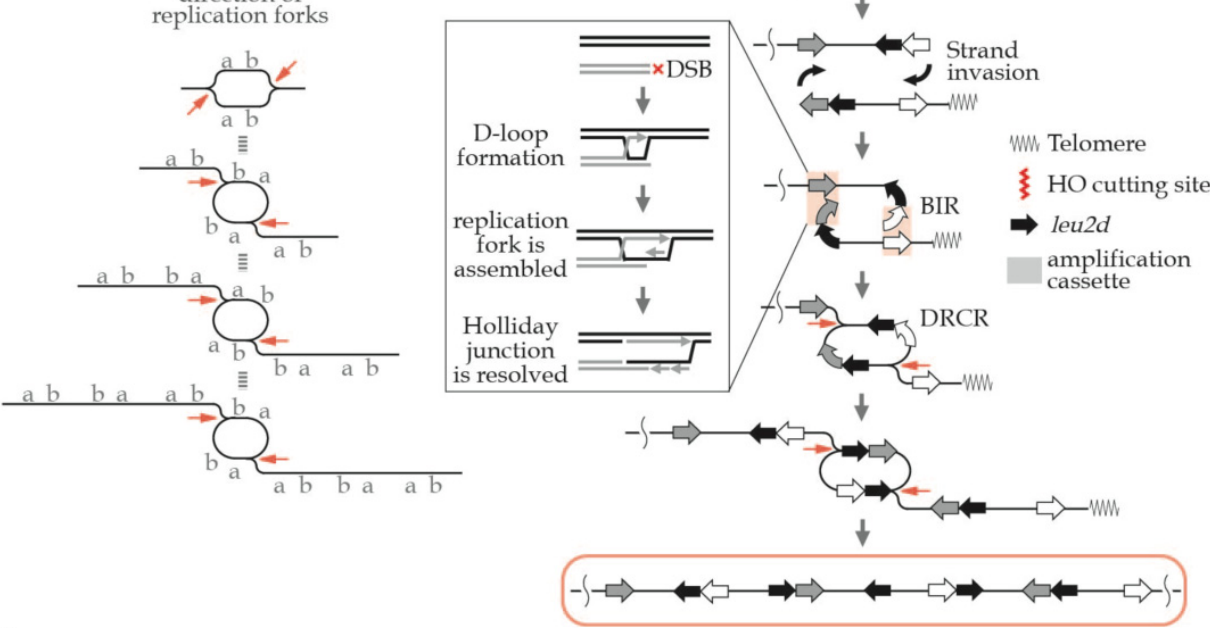

C HSR-type (intra-chromosomal: 100)
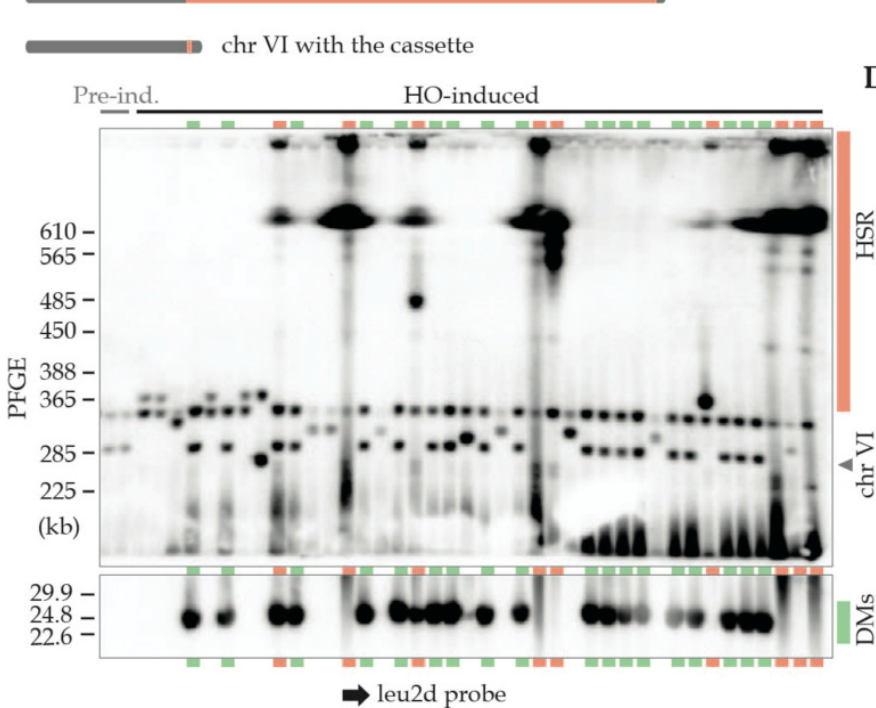

D

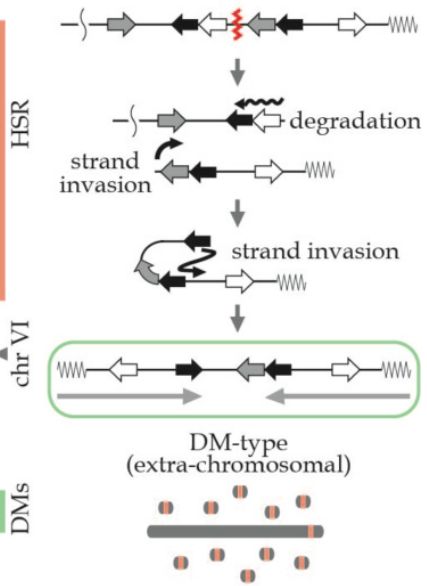

Figure 3. Gene amplification system based on DRCR utilizing break-induced replication (BIR).

(A) Double rolling-circle replication (DRCR). Two replication forks chase each other. One replication fork can replicate a template for the other fork and so amplification proceeds. (B) Structure of the amplification cassette and a model for DRCR amplification. This cassette contains two PCR-amplified sequences (white and gray arrows) derived from the nearby 
genomic region, forming two inverted pairs (a) and (b). The amplification marker, leu2d, has a slight transcription activity, and it will complement leucine auxotrophy if amplified. This yeast strain has galactose-inducible $\mathrm{HO}$ endonuclease gene. Following $\mathrm{HO}$ cutting, two chromosomal ends can invade each other, initiating two break-induced replication (BIR) events as in the insert box and subsequent DRCR. The DRCR process would terminate by recombination between bidirectionally elongated arms. (C) Southern analysis of uncut chromosomal DNA from $\mathrm{Leu}^{+}$survivors with the leu2d probe. The expression of $\mathrm{HO}$ endonuclease was induced on galactose medium without leucine (HO-induced). PFGE was performed with higher and lower size ranges. The lanes marked in red and green indicate intra- and extra-chromosomal amplification, respectively. Pre-ind.: preinduction conditions (cultured on glucose plates containing leucine). (D) Model for the production of extrachromosomal products. These products are proposed to result from degradation of one broken end and the subsequent intramolecular BIR.

\subsection{Replication stress within repeated sequences}

Recently, DNA replication stress within repeated sequences is reported to contribute importantly to genome instability. Two recent yeast papers have shown that nearby inverted repeats recombine spontaneously to fuse, leading to the formation of dicentric and acentric chromosomes (Branzei and Foiani, 2010a; Mizuno et al., 2009; Paek et al., 2009). This fusion process does not appear to require DSB formation, and is likely caused by DNA replication-based mechanism involving an aberrant switch of replication templates.

Another example involves the re-replication event, the inappropriate firing of replication origins. Green et al. developed an elegant system in yeast that enables a locus-specific and transient re-replication by conditionally deregulating the replication origin (Green et al., 2010). They demonstrate that re-replication can generate duplication in cooperation with Ty repetitive elements, suggesting that this process is a potent inducer of gene amplification.

We have examined whether gene amplification can be induced when recombinational processes between inverted sequences are coupled with DNA replication. To efficiently induce the recombinational processes, Cre-lox site-specific recombination was used to design amplification system based on DRCR (Watanabe et al., 2011). This system successfully yielded HSR/DM-type products in yeast (Fig.4) and Chinese hamster ovary (CHO) cells (Fig.5). We first predicted that, if recombination occurs between un-replicated and recently replicated regions during replication (Fig.4A), the replication fork would make an additional copy of the replicated region. To induce DRCR, two sets of the recombinational process were utilized (Fig. $4 \mathrm{~B}$ and $4 \mathrm{C}$ ). In yeast, the Cre induction caused a $>7000$-fold increase in the frequency of survivors and, surprisingly, over $10 \%$ of the Cre recombination-induced cells undergo gene amplification (Fig.4D). The HSR-type products appear to contain approximately 90-140 copies of the leu2d gene, corresponding to a 3.6 5.6fold increase in the length of the original $(275 \mathrm{~kb})$ chromosome VI (Fig.4E). 
For DRCR system in $\mathrm{CHO}$ cells, we constructed an amplification cassette on a rat genomic bacterial artificial chromosome (BAC), and integrated it into a specific site on a CHO cell chromosome using the Flp-FRT (Flp recombination target site) system (Fig.5A). An amplification marker, a mouse dihydrofolate reductase (DHFR) gene, provides methotrexate (MTX) resistance when amplified. This system successfully produced HSR/DM/Scatteredtype amplification (Fig.5B-K).

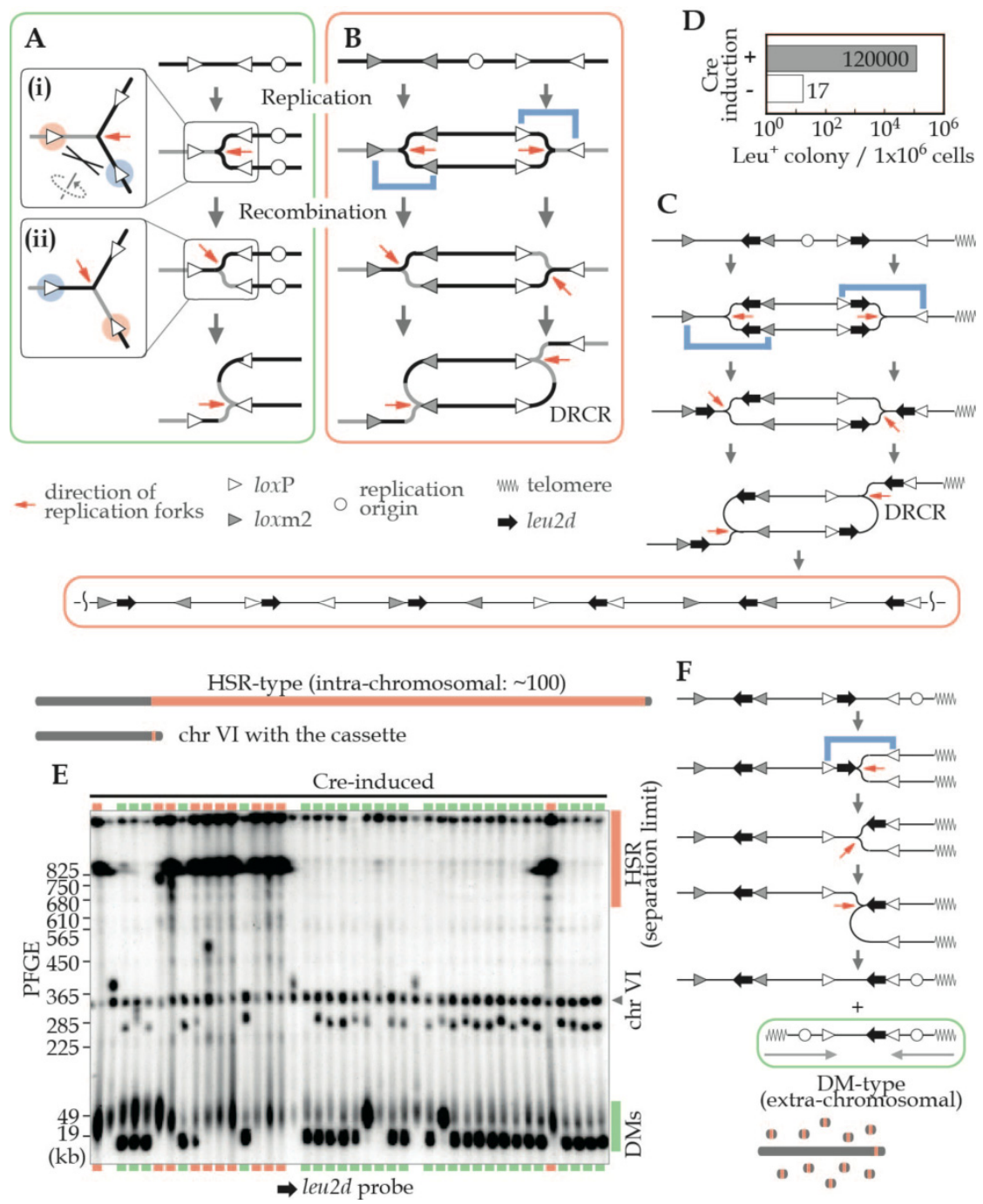

Figure 4. Gene amplification in yeast induced by Cre recombination.

(A) Recombinational process coupled with replication. The gray and black lines indicate the un-replicated and recently-replicated regions at the time of recombination, respectively. If 
recombination occurs between loxP sites marked red and blue (i), the replication template is switched and thereafter the replicated region is replicated again (ii). (B) DRCR induction. If both bidirectional DNA replications undergo the processes as described in (A), DRCR can be induced. Two different types of lox sequence, the wild-type lox $\mathrm{P}$ (lox for short) and a mutant-type loxm2 (m2 for short) were used. Cre recombination occurs between identical sites (lox-lox or m2-m2) but not between different sites (lox-m2). (C) Structure of the amplification cassette and a model for DRCR amplification. (D) Frequency of Leu+ colony formation. (E) Southern analysis of uncut chromosomal DNA from $\mathrm{Leu}^{+}$survivors with the leu $2 d$ probe. The expression of Cre recombinase was induced in galactose medium for 90 min (Cre-induced). PFGE was performed with a wide-size range. The lanes marked in red and green indicate intra- and extra-chromosomal amplification, respectively. (D) Model for the production of extra-chromosomal products. These products are proposed to result from a single recombinational process coupled with DNA replication.

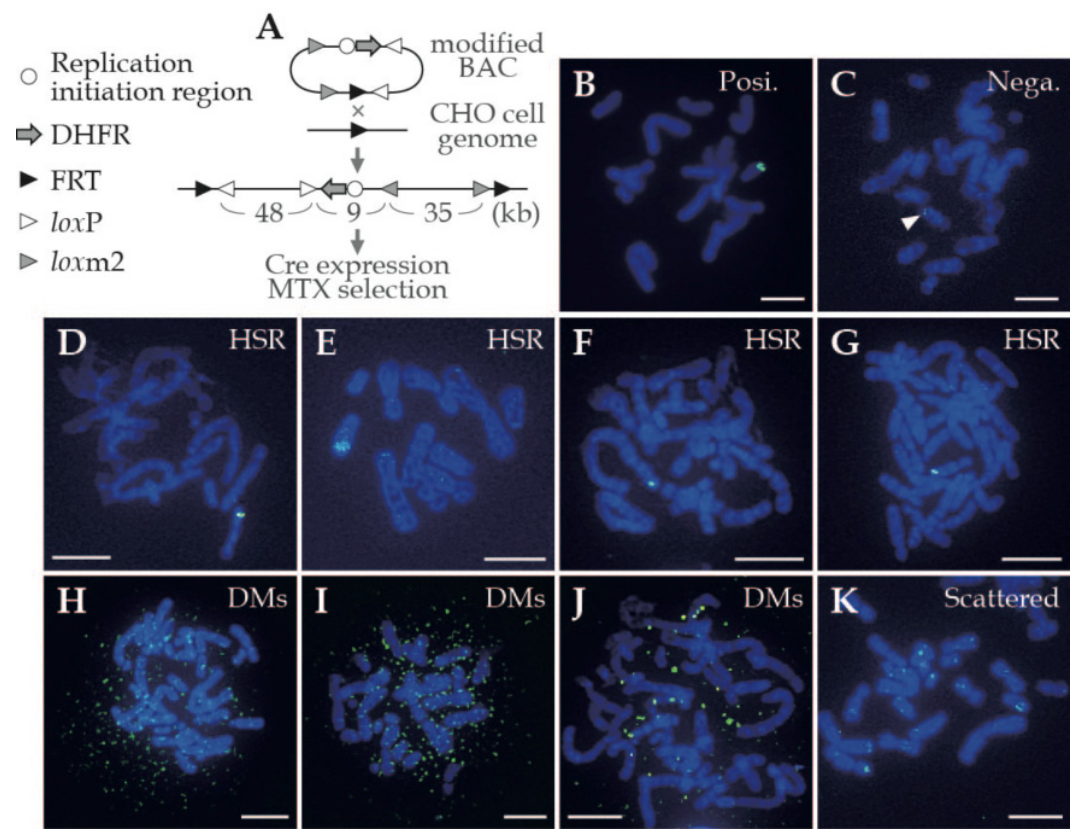

Figure 5. Gene amplification in $\mathrm{CHO}$ cells induced by Cre recombination.

(A) Structure of the modified BAC and construction of the $\mathrm{CHO}$ strain for gene amplification. The sizes $(\mathrm{kb})$ of the three regions in the structure are indicated below. (B) to (K) Metaphase FISH analysis with FITC-labeled probes (green). As a positive control (B), the CHO DR1000L-4N strain that contains $~ 170$ copies of DHFR was probed with a pSV2-dhfr plasmid. The BAC-CHO strain ( $\mathrm{C}$; negative control) without Cre induction and MTX selection and MTX-resistant clones (D-K) were probed with the BAC in (A). DNA is counterstained with DAPI (blue). The scale bars represent $10 \mu \mathrm{m}$. These amplified products would be derived from the integrated BAC construct. 


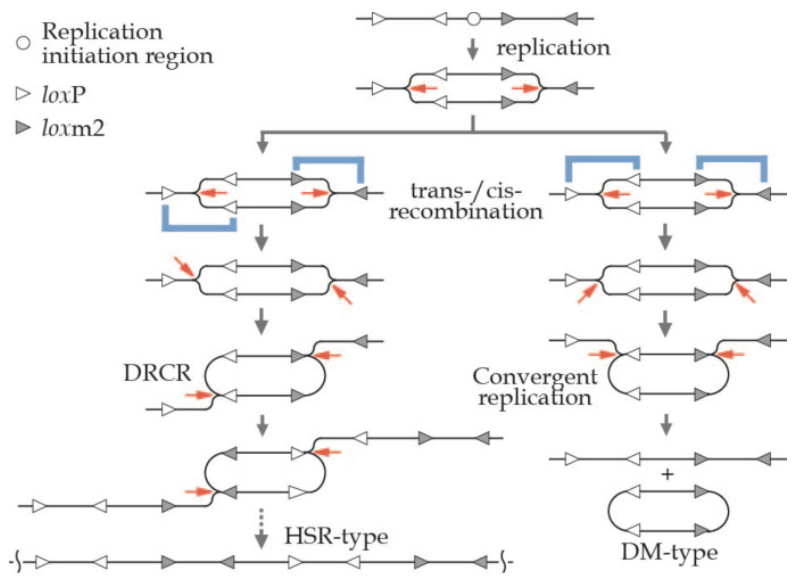

Figure 6. Model for HSR/DM production in the CHO system.

A model for the HSR and DMs production by Cre recombination coupled with replication. See in the text.

Our Cre-lox system can induce tissue-specific amplification, and therefore may allow a direct approach to examine which genetic elements contribute to oncogenesis or malignant potential in each tissue when amplified. In addition, our CHO system showed scatteredtype amplification products resembling those seen in cancer cells, although in noncancerous cell line. From these results, we reasoned that DRCR are centrally involved in amplification of drug-resistance genes and oncogene. This system can serve as a good model for amplification in mammalian cells and contribute to a better understanding of oncogene amplification and development of anticancer strategies in the future.

The formation of HSR/DM-type products can be explained by Cre recombination coupled with replication in two alternative ways, by trans- or cis-recombination, which can induce either DRCR or convergent replication, respectively (Fig.6). The scattered-type amplification may be generated by reintegration of DM-type products into ectopic chromosomes through interspersed repetitive elements. In gene amplification in mammalian cells, BFB cycles would form megabase-sized inverted repeats, which may induce DRCR if homology-based recombination is coupled with DNA replication. Recently, a similar process, replication template exchange, was reported to lead to acentric or dicentric chromosome formation in yeast, indicating an important contribution to genome instability (Branzei and Foiani, 2010a; Mizuno et al., 2009; Paek et al., 2009). We propose that such processes can occur in cultured cells and tumor cells through genome instability associated with deregulated replication (Aguilera and Gomez-Gonzalez, 2008; Branzei and Foiani, 2010b).

\subsection{Rearrangements in amplified regions}

In amplified chromosomal regions, intensive chromosome rearrangements are frequently observed, leading to the increase in the gene copy number and to the decrease in size of 
the amplification unit (Debatisse and Malfor, 2005; Mondello et al., 2010). Nuclear blebs and micronuclei are frequently observed in cells with gene amplification and found to contain amplified sequences and thus may be a location for rearrangement of amplified region. However, how the rearrangements proceed is a long-standing question. In oncogene amplification, the complex patterns of amplification generated by the rearrangements are closely associated with poor prognosis in cancer (Chin et al., 2006; Hicks et al., 2006). Interestingly, we have observed the rearrangement in all our DRCR systems (Fig.7A and 7B). Sequences flanked by inverted repeats, which are formed by DRCR amplification, were subject to frequent inversion. We call this phenomenon DRCRdependent inversion. To explore the link between the rearrangements and the DRCR process, we constructed a system that can turn on or off the occurrence of DRCR, using yeast $2 \mu$ plasmid (Okamoto et al., 2011). This system demonstrated that inversions, deletions, or duplications could be intensively induced in a DRCR-dependent manner. This result suggests that DRCR may cause the rearrangements in amplification in nature.

DRCR-dependent inversion is an interesting phenomenon, but the mechanism remains unknown. DRCR is expected to form an unstable structure, a palindromic structure. We propose that DRCR-dependent inversion may disrupt the palindromic structure and substantially stabilize the highly repetitive array (Fig.7B). We also proposed a model in which DRCR markedly stimulates recombinational events (Fig.7C). In eukaryotes, a protein complex, cohesin, bundles newly replicated sister chromatids until anaphase and regulates the separation of sister chromatids during cell division (Nasmyth, 1999). In DRCR process, however, one of newly replicated sister chromatids is used as a template for another replication fork, and therefore cohesin would fail to bundle the sister chromatids together. These cohesion-free regions are expected to be recombinogenic based on some data indicating activated recombination under cohesion-deficient conditions (GrossenbacherGrunder \& Thuriaux, 1981; Kobayashi et al. 2004).

Recently, a chromosome catastrophe phenomenon termed chromothripsis, in which numerous rearrangements are apparently acquired in one single catastrophic event, was observed in multiple cancers (Liu et al., 2011). The formation of intensive rearrangements has been proposed to involve a replication-based mechanism, the fork stalling and template switching (FoSTeS) model (Lee et al., 2007). The FoSTeS process may be engaged also in the intensive rearrangements in amplified chromosomal regions.

In cancer and drug-resistant cells, BFB cycles form large regular inverted repeats in the early stages of amplification, and thereafter these repeats rapidly change into shorter highly amplified units. However, it remains largely unknown how complex end products can be rapidly generated after BFB cycles. We expect that DRCR process play a key role in linking BFB cycles to complex end products. DRCR process may be initiated by DSBs or DNA replication stress within inverted chromosome regions formed through BFB cycles. This involvement of DRCR is supported by a recent data that HSR was lengthened more rapidly than expected from BFB cycle model (Harada et al., 2011). 
A

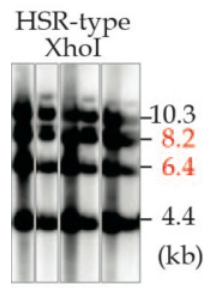

B

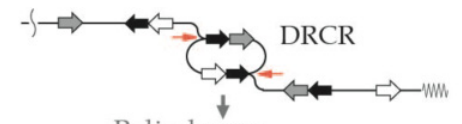

Palindrome

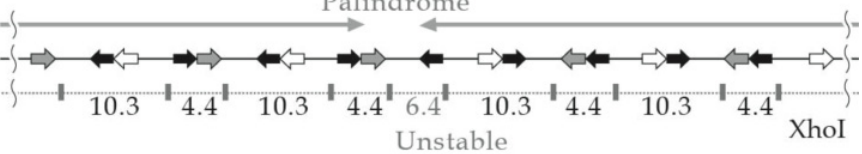

Stable

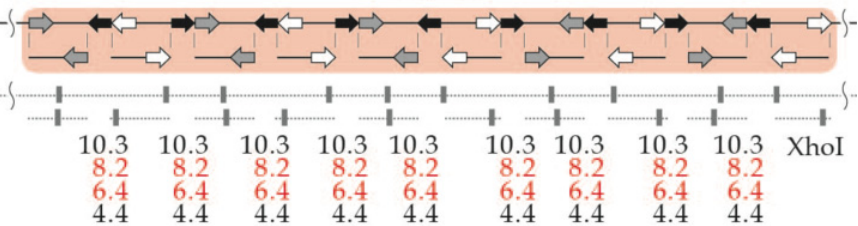

C
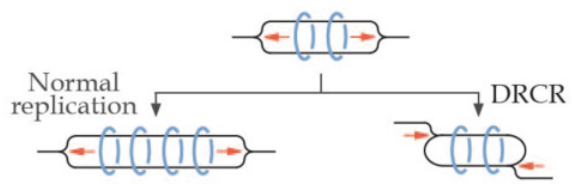

Cohesin

$\rightarrow \begin{aligned} & \text { direction of } \\ & \text { replication forks }\end{aligned}$

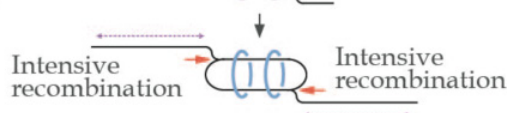

Figure 7. DRCR-dependent rearrangements

(A) Southern analysis of XhoI-digested DNA of some HSR-type samples in our BIR-based DRCR system with the leu2d probe. The fragment sizes in black and red indicate the expected and unexpected band. (B) Schematic representation of the expected structure derived through the DRCR process and XhoI-restriction maps of the representative HSRtype structure. (C) Model of the recombinogenic feature of DRCR. While cohesin complexes bundle newly replicated sister chromatids in normal DNA replication, in DRCR, cohesin would fail to bundle the sister chromatids together, leading to the exposure of recombinogenic region.

\section{Concluding remarks}

The processes of oncogene amplification are difficult to analyze because of the infrequency of amplification and the plasticity of amplified products. The development of model systems is one of the best approaches to overcome the difficulties in elucidating the molecular mechanisms. The model systems can serve as a good model for a better understanding of oncogene amplification and contribute to development of anticancer strategies in future.

Gene amplification is a hallmark of most advanced solid tumors and amplified genes are useful therapeutic targets. Immortalized cells can undergo amplification when selected with appropriate drugs $\left(10^{-4}\right.$ to $\left.10^{-7}\right)$, whereas gene amplification has never been detected in 
normal cells $\left(<10^{-9}\right)$ (Tlsty et al., 1989; Wright et al., 1990). This observation strongly suggests the defect in control of genome integrity in cancer cells.

Furthermore, cancer cells are often dependent on (addicted to) only one or a few genes conferring malignancy and growth advantage, although the cells involve multiple genetic and epigenetic abnormalities (Weinstein and Joe, 2006). This phenomenon, called 'oncogene addiction', is frequently observed with oncogenes associated with amplification, such as MYC, ERBB2, CCND1, and BCR-ABL, indicating that the enhanced expression of amplified genes would become a meaningful therapeutic targets.

The direct involvement of DRCR-related processes in oncogene amplification has yet to be demonstrated. Amplified oncogenes manifest a structural diversity. MYC gene is thought to amplify first as DMs, and thereafter integration into a chromosome can lead to HSR amplification consisting of direct or inverted repeats. Although many tumor cells would undergo BFB cycles, which form inverted array, amplification of MYCN and ERBB2 can be found as HSR with direct tandem repeats (Albertson, 2006). Amplified EGFR genes are present on DMs (Albertson, 2006), and BCR-ABL amplification was found on a chromosome (Gorre et al., 2001). These amplifications could not be explained by only one versatile process, but DRCR-related process may contribute to a variety of oncogene amplification. HSR and DMs in MYC amplification might be produced via DRCR and its related process like convergent replication, respectively (Fig.6). Furthermore, Our system can be adapted to simple rolling-circle replication (RCR) by replacing inverted sequences in our amplification cassettes to direct ones. This RCR forms direct tandem array as seen in amplification of MYCN and ERBB2. The DRCR-related processes can be initiated by any important triggers, such as DSB, inverted repeats, and replication stress, which genome instability in tumor or cancer cells could provide. These trigger reactions may occur via interspersed repetitive elements, including Alus, and short or long interspersed nucleotide element (SINE/LINE). The DRCR-related processes can generate intensive chromosome rearrangement, a common feature of oncogene amplification. Thus, we propose that DRCR-related processes can provide broad contributions to oncogene amplification at multiple phases.

We also believe that optimization and improvement of the model amplification systems could provide benefits for the production of therapeutic proteins. Thus, works that utilize the model systems will have great impact not only on scientific understanding but also in the medical, industrial and economic fields.

\section{Author details}

Takaaki Watanabe

Department of Molecular Genetics, Cleveland Clinic Lerner Research Institute, USA

\section{Acknowledgement}

I am grateful to Prof. Takashi Horiuchi for his supervision in National Institute for Basic Biology (NIBB). I would like to thank all students, technicians, technologist, and faculty who 
have been supporting and guiding me. I appreciate Dr. Hisashi Tanaka giving me helpful discussion in Cleveland Clinic Lerner Research Institute. This was supported by Grant-inAid for Young Scientists (Japan Society for the Promotion of Science); Adaptable and Seamless Technology Transfer Program (Japan Science and Technology Agency); Research Project of the Center for the Promotion of Integrated Sciences of Sokendai.

\section{References}

Aguilera, A and Gomez-Gonzalez, B. 2008. Genome instability: a mechanistic view of its causes and consequences. Nat Rev Genet 9: 204-217.

Albertson, DG. 2006. Gene amplification in cancer. Trends Genet 22: 447-455.

Branzei, D and Foiani, M. 2010a. Leaping forks at inverted repeats. Genes Dev 24: 5-9.

Branzei, D and Foiani, M. 2010b. Maintaining genome stability at the replication fork. Nat Rev Mol Cell Biol 11: 208-219.

Butler, DK, Yasuda, LE, and Yao, MC. 1996. Induction of large DNA palindrome formation in yeast: implications for gene amplification and genome stability in eukaryotes. Cell 87: 1115-1122.

Chin, K, DeVries, S, Fridlyand, J, Spellman, PT, Roydasgupta, R, Kuo, WL, Lapuk, A, Neve, RM, Qian, Z, Ryder, T et al. 2006. Genomic and transcriptional aberrations linked to breast cancer pathophysiologies. Cancer Cell 10: 529-541.

Ciullo, M, Debily, MA, Rozier, L, Autiero, M, Billault, A, Mayau, V, El Marhomy, S, Guardiola, J, Bernheim, A, Coullin, P et al. 2002. Initiation of the breakage-fusion-bridge mechanism through common fragile site activation in human breast cancer cells: the model of PIP gene duplication from a break at FRA7I. Hum Mol Genet 11: 2887-2894.

Coquelle, A, Pipiras, E, Toledo, F, Buttin, G, and Debatisse, M. 1997. Expression of fragile sites triggers intrachromosomal mammalian gene amplification and sets boundaries to early amplicons. Cell 89: 215-225.

Debatisse, M, Le Tallec, B, Letessier, A, Dutrillaux, B, and Brison, O. 2012. Common fragile sites: mechanisms of instability revisited. Trends Genet 28: 22-32.

Debatisse, M and Malfor, B. 2005. Gene amplification mechanisms. in Genome Instability in Cancer Development (ed. E.A. Nigg), pp. 343-361. Springer, Netherlands.

Di Fiore, PP, Pierce, JH, Kraus, MH, Segatto, O, King, CR, and Aaronson, SA. 1987. erbB-2 is a potent oncogene when overexpressed in NIH/3T3 cells. Science 237: 178-182.

Erhart, E and Hollenberg, CP. 1983. The presence of a defective LEU2 gene on $2 \mathrm{mu}$ DNA recombinant plasmids of Saccharomyces cerevisiae is responsible for curing and high copy number. J Bacteriol 156: 625-635.

Glover, TW, Arlt, MF, Casper, AM, and Durkin, SG. 2005. Mechanisms of common fragile site instability. Hum Mol Genet 14 Spec No. 2: R197-205.

Gorre, ME, Mohammed, M, Ellwood, K, Hsu, N, Paquette, R, Rao, PN, and Sawyers, CL. 2001. Clinical resistance to STI-571 cancer therapy caused by BCR-ABL gene mutation or amplification. Science 293: 876-880.

Green, BM, Finn, KJ, and Li, JJ. 2010. Loss of DNA replication control is a potent inducer of gene amplification. Science 329: 943-946. 
Grossenbacher-Grunder, AM, and Thyriaux, P. 1981. Spontaneous and UV-induced recombination in radiation-sensitive mutants of Schizosaccharomyces pombe. Mutat Res 81: 37-48.

Haber, JE and Debatisse, M. 2006. Gene amplification: yeast takes a turn. Cell 125: 1237-1240.

Harada, S, Sekiguchi, N, and Shimizu, N. 2011. Amplification of a plasmid bearing a mammalian replication initiation region in chromosomal and extrachromosomal contexts. Nucleic Acids Res 39: 958-969.

Hellman, A, Zlotorynski, E, Scherer, SW, Cheung, J, Vincent, JB, Smith, DI, Trakhtenbrot, L, and Kerem, B. 2002. A role for common fragile site induction in amplification of human oncogenes. Cancer Cell 1: 89-97.

Hicks, J, Krasnitz, A, Lakshmi, B, Navin, NE, Riggs, M, Leibu, E, Esposito, D, Alexander, J, Troge, J, Grubor, V et al. 2006. Novel patterns of genome rearrangement and their association with survival in breast cancer. Genome research 16: 1465-1479.

Kobayashi, T, Horiuchi, T, Tongaonkar, P, Vu, L, and Nomura, M. 2004. SIR2 regulates recombination between different rDNA repeats, but not recombination within individual rRNA genes in yeast. Cell 117: 441-453.

Kuo, MT, Sen, S, Hittelman, WN, and Hsu, TC. 1998. Chromosomal fragile sites and DNA amplification in drug-resistant cells. Biochem Pharmacol 56: 7-13.

Kuo, MT, Vyas, RC, Jiang, LX, and Hittelman, WN. 1994. Chromosome breakage at a major fragile site associated with P-glycoprotein gene amplification in multidrug-resistant CHO cells. Mol Cell Biol 14: 5202-5211.

Lee, JA, Carvalho, CM, and Lupski, JR. 2007. A DNA replication mechanism for generating nonrecurrent rearrangements associated with genomic disorders. Cell 131: 1235-1247.

Lengauer, C, Kinzler, KW, and Vogelstein, B. 1998. Genetic instabilities in human cancers. Nature 396: 643-649.

Liu, P, Erez, A, Nagamani, SC, Dhar, SU, Kolodziejska, KE, Dharmadhikari, AV, Cooper, ML, Wiszniewska, J, Zhang, F, Withers, MA et al. 2011. Chromosome catastrophes involve replication mechanisms generating complex genomic rearrangements. Cell 146: 889-903.

Lobachev, KS, Gordenin, DA, and Resnick, MA. 2002. The Mre11 complex is required for repair of hairpin-capped double-strand breaks and prevention of chromosome rearrangements. Cell 108: 183-193.

McClintock, B. 1941. The Stability of Broken Ends of Chromosomes in Zea Mays. Genetics 26: 234-282.

Mizuno, K, Lambert, S, Baldacci, G, Murray, JM, and Carr, AM. 2009. Nearby inverted repeats fuse to generate acentric and dicentric palindromic chromosomes by a replication template exchange mechanism. Genes Dev 23: 2876-2886.

Mondello, C, Smirnova, A, and Giulotto, E. 2010. Gene amplification, radiation sensitivity and DNA double-strand breaks. Mutat Res 704: 29-37.

Muller, WJ, Sinn, E, Pattengale, PK, Wallace, R, and Leder, P. 1988. Single-step induction of mammary adenocarcinoma in transgenic mice bearing the activated c-neu oncogene. Cell 54: 105-115. 
Narayanan, V, Mieczkowski, PA, Kim, HM, Petes, TD, and Lobachev, KS. 2006. The pattern of gene amplification is determined by the chromosomal location of hairpin-capped breaks. Cell 125: 1283-1296.

Nasmyth, K. 1999. Separating sister chromatids. Trends Biochem Sci 24: 98-104.

Okamoto, H, Watanabe, TA, and Horiuchi, T. 2011. Double rolling circle replication (DRCR) is recombinogenic. Genes to cells 16: 503-513.

Paek, AL, Kaochar, S, Jones, H, Elezaby, A, Shanks, L, and Weinert, T. 2009. Fusion of nearby inverted repeats by a replication-based mechanism leads to formation of dicentric and acentric chromosomes that cause genome instability in budding yeast. Genes Dev 23: 2861-2875.

Paulson, TG, Almasan, A, Brody, LL, and Wahl, GM. 1998. Gene amplification in a p53deficient cell line requires cell cycle progression under conditions that generate DNA breakage. Mol Cell Biol 18: 3089-3100.

Poupon, MF, Smith, KA, Chernova, OB, Gilbert, C, and Stark, GR. 1996. Inefficient growth arrest in response to dNTP starvation stimulates gene amplification through bridgebreakage-fusion cycles. Mol Biol Cell 7: 345-354.

Rattray, AJ, Shafer, BK, Neelam, B, and Strathern, JN. 2005. A mechanism of palindromic gene amplification in Saccharomyces cerevisiae. Genes Dev 19: 1390-1399.

Savelyeva, L, and Schwab, M. 2001. Amplification of oncogenes revisited: from expression profiling to clinical application. Cancer Lett 167: 115-123.

Slamon, DJ, Clark, GM, Wong, SG, Levin, WJ, Ullrich, A, and McGuire, WL. 1987. Human breast cancer: correlation of relapse and survival with amplification of the HER-2/neu oncogene. Science 235: 177-182.

Tanaka, H, Tapscott, SJ, Trask, BJ, and Yao, MC. 2002. Short inverted repeats initiate gene amplification through the formation of a large DNA palindrome in mammalian cells. Proc Natl Acad Sci U S A 99: 8772-8777.

Tanaka, H and Yao, MC. 2009. Palindromic gene amplification--an evolutionarily conserved role for DNA inverted repeats in the genome. Nat Rev Cancer 9: 216-224.

Tlsty, TD, Margolin, BH, and Lum, K. 1989. Differences in the rates of gene amplification in nontumorigenic and tumorigenic cell lines as measured by Luria-Delbruck fluctuation analysis. Proc Natl Acad Sci U S A 86: 9441-9445.

Tower, J. 2004. Developmental gene amplification and origin regulation. Annu Rev Genet 38: 273-304.

Vogt, N, Lefevre, SH, Apiou, F, Dutrillaux, AM, Cor, A, Leuraud, P, Poupon, MF, Dutrillaux, B, Debatisse, M, and Malfoy, B. 2004. Molecular structure of double-minute chromosomes bearing amplified copies of the epidermal growth factor receptor gene in gliomas. Proc Natl Acad Sci U S A 101: 11368-11373.

Volkert, FC and Broach, JR. 1986. Site-specific recombination promotes plasmid amplification in yeast. Cell 46: 541-550.

Watanabe, T and Horiuchi, T. 2005. A novel gene amplification system in yeast based on double rolling-circle replication. EMBO J 24: 190-198. 
Watanabe, T, Tanabe, H, and Horiuchi, T. 2011. Gene amplification system based on double rolling-circle replication as a model for oncogene-type amplification. Nucleic acids research 39: e106.

Weinstein, IB and Joe, AK. 2006. Mechanisms of Disease: oncogene addiction-a rationale for molecular targeting in cancer therapy. Nature Clinical Practice Oncology 3: 448-457.

Wright, JA, Smith, HS, Watt, FM, Hancock, MC, Hudson, DL, and Stark, GR. 1990. DNA amplification is rare in normal human cells. Proc Natl Acad Sci U S A 87: 1791-1795.

Yunis, JJ, Soreng, AL, and Bowe, AE. 1987. Fragile sites are targets of diverse mutagens and carcinogens. Oncogene 1: 59-69. 